\title{
Inpatient resource utilisation in younger (2-5 yrs) and older (6-14 yrs) asthmatic children in Finland
}

\author{
E. Valovirta*, V. Sazonov Kocevar", M. Kaila , M. Kajosaari ${ }^{+}$, A. Koivikko ${ }^{\S}$, K. Korhonen ${ }^{f}$, \\ O. Linna**, M. Mäkelä ${ }^{\# \#, ~ K . ~ R e m e s ~}{ }^{f}$, R.H. Ben-Joseph ${ }^{\text {ฯ }}$
}

Inpatient resource utilisation in younger (2-5 yrs) and older (6-14 yrs) asthmatic children in Finland. E. Valovirta, V. Sazonov Kocevar, M. Kaila, M. Kajosaari, A. Koivikko, K. Korhonen, O. Linna, M. Mäkelä, K. Remes, R.H. Ben-Joseph. (CERS Journals Ltd 2002.

ABSTRACT: Asthma is the most common chronic disorder among Finnish children, however, the economic burden of paediatric asthma in Finland has not yet been comprehensively evaluated. The objective of this study was to compare inpatient resource utilisation between younger (2-5 yrs) and older children (6-14 yrs) with asthma in Finland.

A national database of inpatient resource utilisation was applied to determine use of hospital services among children with asthma in 1999. Regional estimates of charges were combined with hospitalisation episodes to determine total inpatient cost.

The results indicate that younger asthmatic children consume 3-times more inpatient resources per capita. Incidence of first admissions because of asthma was 3-times higher in younger children. Hospitalisation and rehospitalisation rates were also 3- and 4-times higher, respectively.

The total annual inpatient cost of asthma in children aged 2-5 and 6-14 yrs was $€ 1.98$ million with each group accounting for $€ 1.12$ million and $€ 0.86$ million, respectively. Regional and age-related differences in hospitalisation rates and costs were likely related to variable clinical practice on the primary level, difficulties with diagnosis and compliance among younger children.

Eur Respir J 2002; 20: 397-402.

\begin{abstract}
*Turku Allergy Centre, Turku, Finland. \#Purdue University, School of Pharmacy, Dept of Pharmacy Practice, West Lafayette, Indiana, USA. "Tampere University Hospital and University of Tampere, Dept of Paediatrics, Tampere, ${ }^{+}$University of Helsinki, Dept of Paediatrics, Helsinki, ${ }^{\S}$ Turku University Hospital and Turku University, Dept of Pediatrics, Turku, ${ }^{f}$ University of Kuopio, Dept of Pediatrics, Kuopio, **Oulu University Hospital, Dept of Pediatrics, Oulu, and ${ }^{\#}$ Helsinki University, Skin and Allergy Hospital, Helsinki, Finland. ${ }^{\top}$ Princeton, New Jersey, USA.
\end{abstract}

Correspondence: V. Sazonov Kocevar, Purdue University, Dept of Pharmacy Practice, 1335 R. Heine Pharmacy Building, West Lafayette, IN 47907-1335, USA.

Fax: 17654961886

E-mail: vaska@pharmacy.purdue.edu

Keywords: Asthma, cost, hospitalisation, inpatient, paediatric

Received: April 202001

Accepted after revision: November 20 2001

This study was supported in part by Merck \& Co., Inc., Whitehouse Station, New Jersey, USA.
Asthma is the most common chronic disorder among Finnish children. About 3\% of the overall Finnish population has been diagnosed with asthma and approximately $12 \%$ of asthmatics are $<15$ yrs of age [1]. The prevalence rates of paediatric asthma in Finland reported in the 1990s ranged from 4 to $7 \%$ [2]. Early diagnosis is usually difficult in children, especially because the airflow obstruction and airway hyperresponsiveness are hard to measure, and because preschool children are often unable to do spirometry or even peak flow measures. However, early diagnosis and appropriate drug therapy to treat inflammation are likely to improve prognosis of asthma in both adults and children [1].

Asthma represents a substantial burden on healthcare systems in all industrialised countries studied so far [3-7]. The total annual cost of asthma in Finland was estimated at $\sim € 0.44$ billion in the 1990s [1].
The economic burden of paediatric asthma in Finland has not been evaluated extensively in the past. Evidence has shown that hospitalisations account for more than half of all expenditures related to asthma management [8]. The objective of this study was to compare inpatient resource utilisation between younger children (aged 2-5 yrs) and older children (aged 6-14 yrs) with asthma in Finland.

\section{Methods}

This study was a retrospective, aggregate database analysis where the volume of services involved and their respective unit costs are presented on a regional and national level for both younger (aged 2-5 yrs) and older (aged 6-14 yrs) children with asthma in 1999. 


\section{Volume of services}

Volume of inpatient services was estimated on the basis of the national database provided by the National Research and Development Centre for Welfare and Health (STAKES, Helsinki, Finland). Additionally, statistics on special reimbursement (75\% coverage) for asthmatic children aged 2-5 and 6-14 provided by The Social Insurance Institution (KELA, Finland) were used to estimate the population of asthmatic children in Finland. Children with chronic asthma are entitled to $75 \%$ reimbursement for medication [1]. In order to obtain this reimbursement, each child's physician needs to examine the patient and determine whether the patient qualifies or not. The most important point in the assessment is the severity of the condition as assessed by the physician. It is therefore safe to assume that patients who receive this benefit are those who are most likely to have at least moderate or severe asthma. Literature has shown that severity of asthma can be one of the determinants of high utilisation of resources, since control of the disease may be more difficult to achieve. Therefore, the implicit assumption in the current authors' calculations is that patients with severe asthma are likely to incur most or all of the inpatient services utilisation. Since data on the exact number of children with asthma are not known for Finland except with approximation via (nonage specific) prevalence rates, the authors decided to conservatively estimate the per capita utilisation and use population at high risk for development of asthma exacerbations as a proxy for asthmatic paediatric population. Nationwide and regional analyses were performed after classifying the districts reported in both documents into five University Hospital regions (Greater Helsinki, Turku, Tampere, Kuopio and Oulu), each serving about 1 million inhabitants [9] (Appendix 1).

The STAKES database on hospitalisations, including all hospital districts in Finland in 1999, recorded the following variables for paediatric and adult patients classified as asthmatics (coded as J45-J46 under the International Classification of Diseases (ICD)-10 system): age, number of hospitalised patients, number of treatment days incurred by these patients, number of hospital episodes (including only overnight stay) and number of patients who came for unscheduled visits (i.e. transfers from other hospitals, emergency visits etc.). Additionally, data on the number of patients with special reimbursement for asthma in Finland (per region) as well as the number of all children living in Finnish regions in 1999 (Data obtained from official statistics in Finland) were used for calculation of hospitalisation parameters. In 1999 there were 7,772 children aged 2-5 yrs and 18,157 children aged 6-14 yrs entitled to special reimbursement for asthma (KELA).

Using all three data sources described above, the following parameters were calculated for both age groups: distribution of hospitalisations per regions; hospitalisation rates per 1,000 patients, which incorporates both first and repeated hospitalisations; hospitalisation rates per 1,000 children; age standardised incidence of first admission due to asthma, calculated as the ratio of first admissions per 1,000 children, and rehospitalisation rates, calculated as the ratio of repeated hospitalisations per 1,000 patients, per 1,000 children and per hospitalised children. Incidence of first admissions is defined as first admission during a 1-yr observation period. Additionally, the length of stay (LOS) per episode was calculated in a particular region and nationwide. The latter provided information necessary to estimate the direct resources consumed per hospital episode.

\section{Cost of services}

University hospitals and hospital districts in five major Finnish regions provided cost data. When multiple cost estimates were available from hospital districts with multiple institutions, the average cost per district was used. Charges were used as a proxy for costs in 1999 in all regions except one (Helsinki). In Helsinki charges were based upon the Diagnosis Related Groups (DRG), a patient classification system used to define different types of inpatients. The DRG98 includes cost per episode of treatment for children and teenagers $<18$ yrs of age with respiratory problems, bronchitis and asthma as defined by ICD10 codes. In all the other regions, daily charges and average LOS per episode were combined in order to estimate the regional cost per episode.

The total annual costs $(€)$ of inpatient visits for the two groups of asthmatic children for 1999 were estimated as follows. The number of episodes per region was multiplied by the corresponding cost figures per episode. Additionally, the inpatient cost per asthmatic child on special reimbursement (per capita), and the cost per hospitalised child was estimated. All currency conversions were conducted with the Purchase Power Parity adjusted exchange rate for the corresponding year published by the Organisation of Economic Cooperation and Development (OECD).

\section{Results}

\section{Hospitalisations}

All hospitalisation parameters, except the average LOS, suggested that the younger group of asthmatic patients (2-5 yrs) utilised the inpatient resources more frequently than the older children in 1999 (table 1). Younger children (2-5 yrs) experienced about 3-times higher rate of hospitalisations and a 4-times higher rate of rehospitalisations when compared to older children (6-14 yrs) in Finland. Higher rehospitalisation rates among younger children suggest a lower degree of disease control compared to older children. Nationwide incidence of first admission due to asthma was 3-times greater in the younger group. This parameter often indicates the underlying disease prevalence.

The majority of the 1999 hospitalisations (total of 853 episodes) for asthmatic children between 2-5 yrs in Finland were in the Helsinki region $(28.6 \%)$, closely followed by Kuopio (27.3\%) and Tampere regions (22.6\%). Turku and Oulu regions accounted for $10.5 \%$ 
Table 1. - Summary of 1999 hospitalisation parameters for asthmatic children aged 2-5 and 6-14 yrs in Finland

\begin{tabular}{|c|c|c|}
\hline Hospitalisation parameter & Age group $2-5$ & Age group 6-14 \\
\hline Total patients hospitalised $n$ & 853 & 635 \\
\hline Total hospital days $\mathrm{n}$ & 2237 & 1853 \\
\hline Total hospital episodes $n$ & 1053 & 740 \\
\hline Hospitalisations per 1000 children with asthma & 135.6 & 40.8 \\
\hline Hospitalisations per 1000 children & 4.2 & 1.3 \\
\hline Incidence of first admissions per 1000 children $^{\#}$ & 3.43 & 1.09 \\
\hline Average rehospitalisation rate per 1000 children with asthma & 25.7 & 5.8 \\
\hline Average rehospitalisation rate per 1000 children $^{+}$ & 0.8 & 0.2 \\
\hline Average rehospitalisation per 100 hospitalised children in $1999 \%$ & 23.4 & 16.5 \\
\hline Average (between regions) length of stay per episode days ${ }^{f}$ & 2.1 & 2.5 \\
\hline Average (between regions) length of stay per patient days ${ }^{\ddagger}$ & 2.6 & 2.9 \\
\hline
\end{tabular}

\#: total patients hospitalised $\times 1000 /$ children; $^{\top}$ : (total hospital episodes-total patients hospitalised) $\times 1000 /$ children with asthma; +: (total hospital episodes-total patients hospitalised) $\times 1000 /$ children; $\$$ : (total hospital episodes-total patients hospitalised) $\times 100 /$ total patients hospitalised; ${ }^{f}$ : total hospital days/total hospital episodes; ${ }^{\# \#}$ : total hospital days/total patients hospitalised.

and $11 \%$ of nationwide hospitalisations, respectively. In the older group, the majority of hospitalisations (total of 635 episodes) were recorded in Kuopio region $(30.4 \%)$, followed by Tampere $(25.3 \%)$, Helsinki $(20.5 \%)$, Oulu (16.5\%) and Turku regions (7.3\%). These figures deviate from the distribution of the paediatric population in Finland as well as from the distribution of the asthmatic population estimated via special reimbursement. To enable comparison among regions, hospitalisations have been presented per 1,000 paediatric patients with asthma as well as per 1,000 children living in the region, and so was the age standardised incidence of first admissions due to asthma (table 2). The average LOS per episode and the average LOS per child have also been presented in order to enable comparison of hospital efficiency. Results suggest that children in the Kuopio region were more likely to be admitted to the hospital than elsewhere because the incidences of first admissions were 5.70 and 1.93 for the younger and the older age groups, respectively. Additionally, differences in hospitalisation rates ranged between $50-60 \%$ among the regions, with Kuopio region being the highest in both age groups and Oulu/Turku recording the lowest hospitalisation rates. Age standardised rehospitalisation rates (per 1,000 patients and per 1,000 children) were $40 \%$ higher in the Kuopio region than elsewhere (fig. 1).

Average LOS per episode in the younger group of patients was the longest in the Oulu region (2.4 days),

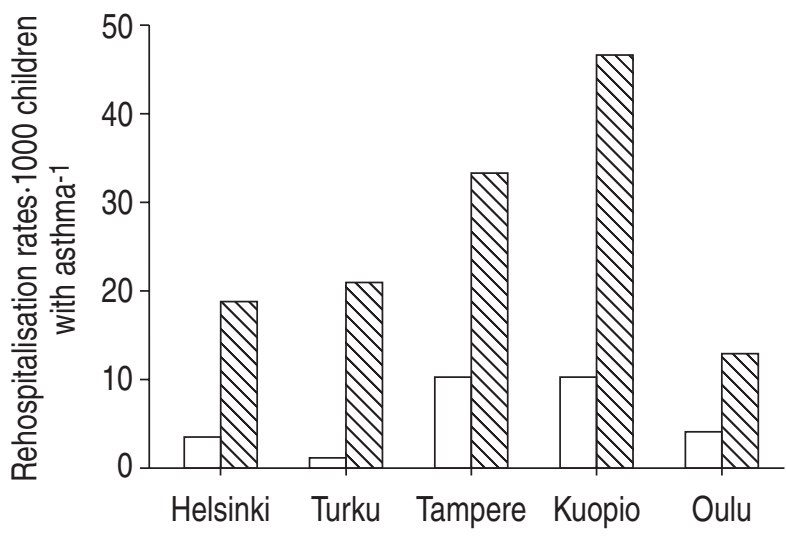

Fig. 1.-1999 Rehospitalisation rates per 1,000 children with asthma in Finnish regions. $\square$ : patients aged $6-14$ yrs; $\mathbb{\mathbb { N }}$ : patients aged $2-5$ yrs.

followed by 2.3 days in Tampere, 2.2 days in Helsinki, 2.1 days in Turku and 1.7 days in the Kuopio region. In the older group, the distribution was exactly the same, however the daily figures were somewhat higher ranging from 2-3 days in all regions (table 2).

\section{Total inpatient costs}

The total annual costs of inpatient visits for asthmatic children aged 2-5 $(\mathrm{n}=853)$ and 6-14 $(\mathrm{n}=635)$ in Finland were $€ 1.12$ million and $€ 0.86$

Table 2. - 1999 Hospitalisations/admissions in Finnish regions

\begin{tabular}{|c|c|c|c|c|c|c|c|c|c|c|}
\hline \multirow[t]{2}{*}{ Age group yrs } & \multicolumn{2}{|c|}{ Helsinki } & \multicolumn{2}{|c|}{ Turku } & \multicolumn{2}{|c|}{ Tampere } & \multicolumn{2}{|c|}{ Kuopio } & \multicolumn{2}{|c|}{ Oulu } \\
\hline & $2-5$ & $6-14$ & $2-5$ & $6-14$ & $2-5$ & $6-14$ & $2-5$ & $6-14$ & $2-5$ & $6-14$ \\
\hline $\begin{array}{l}\text { Hospitalisations per } 1000 \\
\text { patients with asthma }\end{array}$ & 106.4 & 28.1 & 116.9 & 22.6 & 174.5 & 57.4 & 202.2 & 60.2 & 95.7 & 36.3 \\
\hline $\begin{array}{l}\text { Hospitalisations } \\
\text { per } 1000 \text { children }\end{array}$ & 3.6 & 0.8 & 3.4 & 0.7 & 4.3 & 1.4 & 7.4 & 2.3 & 3.1 & 1.3 \\
\hline $\begin{array}{l}\text { Incidence of first admissions } \\
\text { per } 1000 \text { children }\end{array}$ & 2.96 & 0.73 & 2.80 & 0.68 & 3.46 & 1.17 & 5.70 & 1.93 & 2.67 & 1.20 \\
\hline $\begin{array}{l}\text { Average length of stay } \\
\text { per episode (per patient) }\end{array}$ & $\begin{array}{l}2.2 \\
(2.7)\end{array}$ & $\begin{array}{l}2.6 \\
(2.9)\end{array}$ & $\begin{array}{l}2.1 \\
(2.5)\end{array}$ & $\begin{array}{l}2.5 \\
(2.7)\end{array}$ & $\begin{array}{l}2.3 \\
(2.9)\end{array}$ & $\begin{array}{l}2.7 \\
(3.3)\end{array}$ & $\begin{array}{l}1.7 \\
(2.3)\end{array}$ & $\begin{array}{l}2.0 \\
(2.4)\end{array}$ & $\begin{array}{l}2.4 \\
(2.8)\end{array}$ & $\begin{array}{c}3.0 \\
(3.3)\end{array}$ \\
\hline
\end{tabular}


million, respectively. Table 3 summarises the distribution of hospital episodes among regions, the number of patients hospitalised, cost per episode and the total annual costs for the two age groups. The costs per episode differ between the two groups due to differences in LOS per episode.

The inpatient cost per older hospitalised child in 1999 was $€ 1,352$, and $€ 1,320$ per hospitalised young child. Inpatient costs per asthmatic child aged 2-5 and 6-14 with special reimbursement in Finland were estimated at $€ 145$ and $€ 47$, respectively. These results suggest 3-times higher per capita utilisation in the younger group, assuming children on special reimbursement incurred all inpatient costs.

Regional analysis revealed the highest annual inpatient cost per hospitalised young child (2-5 years) in the Helsinki region $€ 1,627$ and the lowest in the Turku region $€ 855$. In the older age group (6-14 yrs), the highest cost per hospitalised child was recorded in the Tampere region $€ 1,528$ and the lowest again in the Turku region $€ 899$. Annual cost per asthmatic child on special reimbursement aged 2-5 was the highest in the Kuopio region $€ 194$ and the lowest in the Turku region $€ 82$. In the older group the annual cost per asthmatic child on special reimbursement was the highest in the Tampere region $€ 72$ and the lowest in the Turku region $€ 20$. As the results suggest, large discrepancies in per capita utilisation among regions exist regardless of the age group, and Turku region showed the lowest per capita utilisation overall. In the long run, marginally higher cost per hospitalised child and longer LOS per episode as well as per child (i.e. Helsinki versus Kuopio region) may be cost saving in terms of overall per capita utilisation. Frequent rehospitalisations may also become more burdensome for parents and children than marginally longer, less frequent and a little bit more expensive hospitalisations. Ultimately more resources are consumed for the same number of patients in the long run with higher frequency and shorter LOS. Alternative explanations must be considered such as differences in disease severity, treatment practice and disease control on the primary care level.

\section{Discussion}

The results presented here suggest that younger asthmatic children (2-5 yrs) in Finland consumed 3-times more inpatient resources per capita (€ 145 versus $€ 47)$ compared to older children (6-14 yrs), both on special reimbursement. Thirty-four per cent more younger patients were hospitalised in 1999, requiring $313(42 \%)$ more hospitalisations (episodes). Younger patients also incurred 21\% more hospital days when compared to the older asthmatic children $(2,237$ versus 1,853$)$ even though the size of the older asthmatic population was greater. Incidence of first admissions because of asthma was 3-times higher in younger children, hospitalisation and rehospitalisation rates in the same age group were also 3-and 4-times higher, respectively. Incidence of first admissions in the older group was comparable with the 1993 data from Denmark [10]. These results suggest that more of the younger than of the older population is admitted and re-admitted to the hospitals because of asthma.

Comparison of findings with other studies is limited because of lack of data on the paediatric burden of asthma. No published studies on the paediatric burden of asthma in Finland are available. Previous results show that LOS due to asthma varies among University Hospitals, which in part explains the variation in inpatient costs [11]. A Swiss study [5] reported the annual inpatient cost per child as Swiss Franc (CHF) 299 (US \$ 150) in 1997. This figure was estimated for 117 asthmatic children $>7$ yrs of age. It is comparable to the per capita consumption in $2-5$ yr old asthmatic children in Finland (US \$ 133), however, when compared to the 6-14 yr age group the costs appear to be much higher in Switzerland. This may be due to the difference in healthcare systems (public/private mix), methodology, data sources, as well as different Gross Domestic Product per capita in the two countries.

A Swedish regional study [12] reported a significant decrease in hospital days and admissions among asthmatic patients aged 5-18 yrs, however there was no such decrease among patients aged 2-4 yrs between 1994 and 1998. The absolute difference between the two groups was observed in terms of hospital days, admissions as well as the total number of patients using the services. The younger population experienced higher utilisation rates (number of hospital days and admissions) in 1998. Perhaps comparison with Sweden is also more appropriate because of the geographical vicinity and similar healthcare system/ organisation. It would be interesting to compare

Table 3. - Inpatient resource utilisation for children aged 2-5 and 6-14 yrs in 1999

\begin{tabular}{|c|c|c|c|c|c|c|c|c|}
\hline & \multicolumn{2}{|c|}{$\begin{array}{l}\text { Hospitalisation } \\
\text { episodes (patients) }\end{array}$} & \multicolumn{2}{|c|}{$\begin{array}{l}\text { Cost per } \\
\text { episode } €\end{array}$} & \multicolumn{2}{|c|}{$\begin{array}{l}\text { Total inpatient costs } \\
\text { in } 1999 \text { Million } €\end{array}$} & \multicolumn{2}{|c|}{$\begin{array}{l}\text { Asthma patients on } \\
\text { special reimbursement }\end{array}$} \\
\hline & $2-5$ & $6-14$ & $2-5$ & $6-14$ & $2-5$ & $6-14$ & $2-5$ & $6-14$ \\
\hline Helsinki & $301(250)$ & $152(135)$ & 1351 & 1351 & 0.41 & 0.21 & 2828 & 5406 \\
\hline Turku & $111(91)$ & $54(51)$ & 700 & 848 & 0.08 & 0.05 & 949 & 2393 \\
\hline Tampere & $238(192)$ & $187(153)$ & 1099 & 1250 & 0.26 & 0.23 & 1364 & 3261 \\
\hline Kuopio & $287(220)$ & 225 (186) & 957 & 1075 & 0.27 & 0.24 & 1419 & 3736 \\
\hline Oulu & $116(100)$ & $122(110)$ & 900 & 1083 & 0.10 & 0.13 & 1212 & 3361 \\
\hline Finland & $1053(853)$ & $740(635)$ & $1069^{\#}$ & $1160^{\#}$ & 1.12 & 0.86 & 7772 & 18157 \\
\hline
\end{tabular}

Purchase Power Parity adjusted exchange rate for 1999 from Organisation of Economic Cooperation and Development data was used as $€$. \#: mean cost per episode in the five cities. 
paediatric inpatient costs between Sweden and Finland, however only figures for adults have been reported in Sweden [6, 7].

A long-term retrospective analysis of hospital admissions for childhood asthma in Finland [13] had similar results, with a relative decrease in acute asthma admissions compared to the number of new asthma medication reimbursements in older children, whereas in the youngest age groups the admissions kept increasing with increasing number of asthma diagnoses. The results of these studies are consistent with the current authors' findings in Finland and suggest higher utilisation of inpatient services among younger asthmatic patients. One possible explanation is that younger children are more prone to viral infections, a major cause for hospital admissions for asthma.

A significant amount of money is still spent on hospital treatment of acute childhood asthma. There is major variation in the diagnostic activity within the country, and recently this difference has been documented between Tampere and Turku in preschool children [14]. The results presented here also suggest that there are high discrepancies in hospitalisation parameters and cost per capita among regions. Kuopio region had 16\% higher hospitalisation rates adjusted for the number of asthmatic patients per region, the highest incidence of first admissions as well as up to $40 \%$ higher rehospitalisation rates in the younger group. Conversely, the LOS was the shortest in the Kuopio region regardless of the age group. One explanation may be the long distances in the eastern part of Finland, which could result in more frequent, but shorter admissions (Appendix 1). However, one would also expect this to be reflected in the Oulu region and in both age groups. Overall, the Kuopio region showed the highest per capita inpatient costs in the younger group, which may suggest that a marginally longer LOS per episode as shown in other regions may decrease the number of rehospitalisations and overall costs.

Other sectors within healthcare associated with treatment of childhood asthma were not studied but could perhaps help explain large variations in inpatient utilisation between regions. It might be the case that in some regions resources consumed for paediatric asthma in other sectors decrease due to larger utilisation in the inpatient sector. In the southern parts of Finland the availability of ambulatory care is greatly improved by specialist care being provided by private practitioners. Hence, access may play an important role in the choice of provider type. Conversely, it has been shown that intensive care accounts for $<1 \%$ of hospital admissions for childhood asthma in Finland [15], although all University hospitals are well equipped for intensive care treatment of childhood asthma. Since data on unscheduled outpatient visits and emergency room visits were not available from registries the effect of these services could not be evaluated in the context of this study.

Traditionally, the diagnosis of asthma has been made reluctantly in young children, and only after at least two documented episodes of wheezing. Contemporary asthma management places focus on prophylactic therapy, and inhaled corticosteroids are the mainstay [1]. It has, however, not been shown conclusively that asthma in childhood is a similar inflammatory condition as has been shown in adults, and evidence to the contrary has actually been presented [16]. Inhaled therapy is technically demanding and complicated delivery systems may not achieve desired effects in paediatric patients $[4,17]$. Therefore, together with new findings in pathophysiology, new treatment options are being sought. Some of the novel drug therapies offered include oral preparations, which may prove useful in improving compliance [17]. However, it has to be emphasised that in small children, the main problem is in the difficulty of making the correct diagnosis, and thereafter adequate instructions on how to use the inhaler or comply with oral therapy are required.

Several limitations are associated with the analyses in this study. Data on children with special reimbursement for asthma, not all asthmatic children in Finland, served as a basis for estimation of hospitalisation rates and inpatient costs per child. As only asthmatic children granted the special reimbursement were included in the analyses, the results of the younger age group may be influenced as the diagnosis of asthma is not made as quickly as in the older age group. Therefore, some estimates may bias the results upwards (hospitalisation rates or inpatient cost per child). Charges were used as a proxy for costs, which is another limitation of the study. The inpatient cost data is therefore not weighted for severity/case mix and is not asthma specific, except for the Helsinki region. This may, in part, explain higher cost per hospitalised child in the Helsinki region. Additionally, a high variation in charges was observed among the sources within the Helsinki region, as well as differences in the description of inpatient cost classes in other regions, both likely consequences of the small area variation phenomenon [18, 19]. Other than the difference in diagnostic activity, all limitations applied to both age groups and therefore the relationship between the two groups should be relatively accurate. It may also be noted that Finnish registries have been shown to be very reliable for healthcare research [20]. Limitation in itself represents the retrospective design of the study as well as the use of aggregate data, which prevents estimation of confidence intervals for some variables of interest (i.e. LOS).

Future research in this area should try to estimate the number of asthmatic patients living in Finland more accurately and also use the DRG standardised cost estimates as soon as they become available. Those improvements would make the results more reliable, as would the longitudinal study design, which would enable a trend analysis. Future focus on assessment of outpatient resource utilisation would also be welcome, since policy decisions in the past might have caused a shift in the use of resources from the inpatient to the outpatient sector.

This study has shown that the total annual cost of asthma inpatient management in Finnish children aged $2-5$ and $6-14$ yrs was $€ 1.98$ million in 1999, each of the groups accounted for $€ 1.12$ million and $€ 0.86$ million, or 57 and $43 \%$ respectively. According to the authors' findings, younger asthmatic children 
(2-5 yrs) use 3-times more of the inpatient resources per capita compared to older asthmatic children (6-14 yrs) in Finland. A reduction of asthma-related complications through improved asthma management might reduce the high hospitalisation rates and resources used, require less physician visits and improve developmental prognosis for young asthmatic patients as well as prove significant for the Finnish healthcare budget.

\section{Appendix}

The map of Finland illustrates the University hospitals in Helsinki, Kuopio, Tampere, Turku and Oulu. Helsinki, Tampere and Turku are situated in the southern part of Finland and serve a greater part of the population. Kuopio in the eastern part and Oulu in the northern part are characterised by long distances.

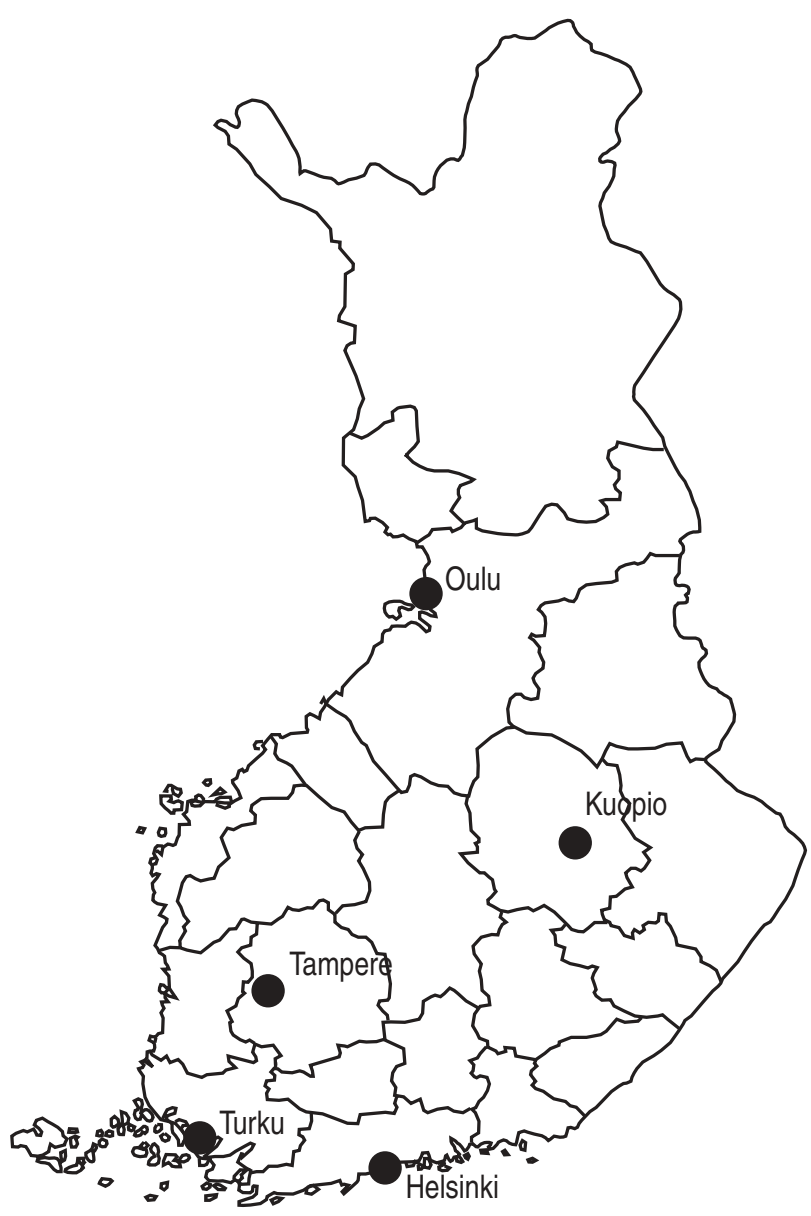

Acknowledgements. The authors would like to thank P. Csonka for his support.

\section{References}

1. Haahtela T, Laitinen LA. Asthma Programme in Finland 1994-2004. Report of a Working Group. Clin Exp Allergy 1996; 26: Suppl. 1, 1-24.
2. Pekkanen J, Remes ST, Husman T, et al. Prevalence of asthma symptoms in video and written questionnaires among children in four regions of Finland. Eur Respir J 1997; 10: 1787-1794.

3. Weiss KB, Gergen PJ, Hodgson TA. An economic evaluation of asthma in the United States. $N$ Engl $J$ Med 1992; 326: 862-866.

4. Barnes PJ, Jonsson B, Klim JB. The costs of asthma. Eur Respir J 1996; 9: 636-642.

5. Szucs TD, Anderhub H, Ruithauser M. The economic burden of asthma: direct and indirect costs in Switzerland. Eur Respir J 1999; 13: 281-286.

6. Thompson S. On the social cost of asthma. Eur J Resp Dis 1984; 65: 185-191.

7. Jacobson L, Lindgren B. Asthma. The socio-economic costs. Studies in economics. Lund, Sweden, Lund University, 1995.

8. Smith DH, Malone DC, Lawson KA, et al. A national estimate of the economic costs of asthma. Am J Respir Crit Care Med 1997; 156: 787-793.

9. Koivusalo M. Decentralisation and equity of healthcare provision in Finland. BMJ 1999; 318: 1198-1200.

10. Bisgaard $\mathrm{H}$, Moller $\mathrm{H}$. Changes in risk of hospital readmission among asthmatic children in Denmark, 1978-93. BMJ 1999; 319: 229-230.

11. Csonka P, Mertsola J, Klaukka T, Kaila M, Ashorn P. Corticosteroid therapy and need for hospital care in wheezing preschool children. Eur J Clin Pharmacol 2000; 56: 591-596.

12. Wennergren G, Strannegard I-L. Continued decrease in hospitalization for asthma in school children but not in young children with increased use of inhaled corticosteroids (Abstract). J Allergy Clin Immunol 1999; 103: S63.

13. Malmström K, Korhonen K, Kaila M, et al. Acute childhood asthma in Finland: a retrospective review of hospital admissions from 1976 to 1995. Pediatr Allergy Immunol 2000; 11: 236-240.

14. Csonka P, Mertsola J, Kaila M, Ashorn P. Regional variation in the diagnosis of asthma among preschool children. Pediatr Allergy Immunol 2000; 11: 189-192.

15. Malmström K, Kaila M, Korhonen K, et al. Mechanical ventilation in children with severe asthma. Pediatr Pulmol 2001; 31: 405-411.

16. Stevenson EC, Turner G, Heaney LG, et al. Bronchoalveolar lavage findings suggest two different forms of childhood asthma. Clin Exper Allergy 1997; 27: 10271035.

17. Williams B, Noonan G, Reiss TF, et al. Long-term asthma control with oral montelukast and inhaled beclomethasone for adults and children 6 years and older. Clin Exp Allergy 2001; 31: 845-854.

18. Physician uncertainty and small area variation. In: Folland S, Goodman AC, Stano M, eds. The economics of health and healthcare, 2nd Edn. Upper Saddle River, NJ, Prentice Hall, 1997.

19. Wennberg J. The Dartmouth atlas of health care 1996, 1998 and 1999. Center for the Evaluative Clinical Sciences at Dartmouth Medical School. www.dartmouthatlas.org. Date last updated: continuous. Date last accessed: 2 September 2001.

20. Gissler M. Administrative registers in health research. A cohort study of Finnish children born in 1987. STAKES, National Research Centre for Welfare and Health. Research Report 97. Helsinki, Finland, STAKES, 1999. 